\title{
Estimating the cancer risk and mortalities induced by routine digital radiography examinations on patient of different ages in Mazandaran province
}

\author{
S.M. Hosseini ${ }^{1}$, A. Banaei ${ }^{2}$, Z. Hoseini Motlagh ${ }^{3}$, R. Abedi-Firouzjah4,5*, \\ F. Falahatí, H. Zamani', Y. Moghimi ${ }^{*}$ \\ ${ }^{1}$ Department of Medical Physics, Faculty of Medicine, Mashhad University of Medical Sciences, Mashhad, Iran \\ ${ }^{2}$ Department of Medical Physics, Faculty of Medical Sciences, Tarbiat Modares University, Tehran, Iran \\ ${ }^{3}$ Babol University of Medical Sciences, Babol, Iran \\ ${ }^{4}$ Department of Medical Physics Radiobiology and Radiation Protection, School of Medicine, Babol University of \\ Medical Sciences, Babol, Iran \\ ${ }^{5}$ Cellular and Molecular Research Center, Yasuj University of Medical Sciences, Yasuj, Iran \\ ${ }^{6}$ Department of Medical Physics, Faculty of Medicine, Shahid Sadoughi University of Medical Sciences, Yazd, Iran
}

\section{- Original article}

\section{*Corresponding authors:}

Dr. Razzagh Abedi-Firouzjah,

Dr. Yousef Moghimi,

E-mail:

r.abedi@mubabol.ac.ir

umoghimi@gmail.com

Revised: june 2020

Accepted: July 2020

Int. J. Radiat. Res., October 2020; 18(4): $875-884$

DOI: 10.18869 /acadpub.ijrr.18.4.853

\begin{abstract}
Background: The aim of this study was to estimate the cancer risks and mortalities of different types induced by routine examinations of digital radiography for one year in Mazandaran province, Iran. Materials and Methods: Radiation parameters and calculated entrance skin dose (ESD) values of 13 digital radiographic examinations were collected from 2340 patients at 18 high-patient-load radiography centers. Organ mean doses were estimated based on the collected parameters applying PCXMC software. The BEIR VII-Phase 2 model was used to calculate the induced cancer risks and mortalities of various cancer types at different ages. Results: The average \pm standard deviation (SD) lifetime risks (incidence probability in 100,000 people) induced by radiations from radiography examinations for one year was $51.29 \pm 4.73$ and $99.62 \pm 7.36$ for new-born males and females, respectively. The lifetime cancer risk decreased with age and reached $3.77 \pm 0.62$ and $4.88 \pm 0.07$ for 80 -year men and women, respectively. The average lifetime risks of mortality due to cancers induced by annual radiographies were obtained at $14.18 \pm 1.62$ and $22.83 \pm 2.55$ for new-born males and females, respectively. This risk reduced with age and was reached $1.97 \pm 0.27$ and $2.45 \pm 0.38$ for men and women at the age of 80 years, respectively. Conclusion: Our results showed that there are low but significant risks of cancer incidence for patients undergoing digital radiography, which included a large percentage of the population in Mazandaran province, especially for children and newborns. Therefore, further efforts like appropriate patient setup and beam geometry should be carried out to decrease patient doses.
\end{abstract}

Keywords: Digital radiography, cancer risk, cancer mortality, Mazandaran provience

\section{INTRODUCTION}

Applying the medical systems which use ionizing radiations is increasing for diagnosis and treatment of different diseases (1-4). Radiography is still one of the medical diagnostic methods besides the new imaging methods such as MRI and CT scans (5). 
Nowadays, improvements in radiology have been made to reduce the radiation dose from $\mathrm{X}$-ray imaging and increase the image quality, therefore, the use of digital radiography systems has become more widespread in the medical examinations than analog radiographies (6). In addition, digital method reduces the number of $\mathrm{X}$ -ray retakes from $5.5 \%$ for analog to $1.0 \%$ (7). Naturally, ionizing radiation from X-ray increases the probability of adverse health issues. Also, it leads to a breakage in molecular bonding in humans and affects chromosome to induce different cancers ${ }^{(8)}$.

Estimating the risk of cancer and preparing the way for reducing this problem is an important issue. In diagnostic radiology examinations, the organ-absorbed dose is used to estimate the cancer risk and hereditary effects to provide effective protection to the patients $(9$, 10).

Entrance surface dose (ESD) is the absorbed dose to the entrance skin of the patient at the central point of the irradiated area and it is used to determine the patient's effective dose $(4,11)$. The patient's effective dose also depends on the $\mathrm{X}$-ray's penetrating power and the body region being examined (12).

Investigation of the cancer risk and mortalities for every diagnostic imaging modality such as digital radiography for every geographical region is essential. This information can be beneficial for the patients' radiation safety in the medical imaging process. There are several studies, investigating the patients' effective dose and cancer risk from diagnostic imaging procedures (13-27). Some of these studies have evaluated and assessed the patient's effective dose alone or with image quality parameters $(14-16,22,23)$, and some others have tried to introduce diagnostic reference levels (DRLs) (24). There are also several studies calculating the risk of cancer depending on the radiology technique $(8,17,18,20,21)$, like oral and panoramic radiographies (21), angiographies (8), or common radiographies from head, chest, and abdomen (18) in different areas. However, the BEIR VII-phase2 (Biological Effects of Ionizing Radiation VII-phase2) has rarely been used for cancer risk estimation. Furthermore, the assessment of cancer risks induced by radiological imaging must be performed in different geographic regions (like different provinces) and various time periods to enable specialists to make better and more accurate estimations of health risks and establish local DRLs.

In the present study, the authors have investigated the induced cancer incident risks and mortalities in Mazandaran province, Iran, from annual digital radiography examinations for different patients' ages and genders. It is notable that the current study is the first research investigating the cancer risk and mortalities in digital radiography examinations in Mazandaran province based on the BEIR VII-Phase 2 model.

\section{MATERIALS AND METHODS}

\section{Data collection}

The present cross-sectional and multi-center study was carried out from 2018-2019 on the data of 2340 patients (1237 males and 1103 females) referring to 18 public and private high-patient-load radiography centers. The sample size was determined based on previous studies with $5 \%$ uncertainty $(28,29)$.

The physical and demographic information of the patients, including the average weight, height, BMI, and thickness of the organs examined in this study is shown in table 1 . The $\mathrm{kVp}, \mathrm{mAs}$, FDD, and FSD values are shown in table 2. The filter thickness values were chosen to $1.5 \mathrm{~mm}-\mathrm{Al}$ for all examinations according to the recommendation of IAEA report for optimization of the radiological protection of patients undergoing digital radiography (30).

The name of the cities and radiology centers include; Babol (Shahid Beheshti, Yahyanejad, and Rouhani hospitals; Mehregan Shomal, and Partov Medical centers), Babolsar (Aryan center), Ghaemshahr (Razi hospital), Sari (Partovmazand center; Bouali Sina and Emam Khomeini [2 systems] hospitals), Amol (Shomal and Emam Reza hospitals), Chalos (Taleghani hospital; Eslami, and Rad centers), and Tonekabon (Shahid Rajaei hospital). Five 
stationary X-ray including a Phillips (Netherlands), Siemens (Germany), Shimadzu (Japan), Toshiba (Japan), and Varian (USA) machines were used. It is notable that all of the radiography systems were calibrated and the quality control procedures were performed in accordance with the American Association of Medical Physics (AAMP) report no. 4 and 74 (31, 32).

Thirteen digital radiographic examinations including skull (PA [posterior-anterior] and LAT [lateral]), cervical (AP [anterior-posterior] and LAT), thoracic (AP and LAT), lumbar (AP and LAT), chest (PA and LAT), abdomen (AP), pelvis (AP), and hip (AP) were selected based on their higher frequency and contribution. The indirect dosimetry method was performed with regard to the IAEA report series no. 457 (33), and 10 patients with standard size based on this report were chosen for each examination. For the patients' dose assessment, ESD (mGy) was calculated.

Table 1. Mean and standard deviation of patient anatomical information.

\begin{tabular}{|c|c|c|c|c|c|c|}
\hline & Number of patient (sex) & Age (years) & Weight $(\mathbf{k g})$ & Height $(\mathbf{c m})$ & BMI $\left(\mathbf{k g} / \mathbf{c m}^{2}\right)$ & Organ thickness (cm) \\
\hline Skull (PA) & $180(101 \mathrm{M}, 79 \mathrm{~F})$ & $45.3 \pm 22.2$ & $68.6 \pm 5.9$ & $166.1 \pm 10.5$ & $26.1 \pm 2.7$ & $11.6 \pm 0.8$ \\
\hline Skull (LAT) & $180(109 \mathrm{M}, 71 \mathrm{~F})$ & $51.7 \pm 28.7$ & $67.7 \pm 5.4$ & $164 \pm 19.6$ & $26.1 \pm 2.3$ & $12.5 \pm 2$ \\
\hline Cervical (AP) & $180(83 \mathrm{M}, 97 \mathrm{~F})$ & $39.8 \pm 18.4$ & $71.7 \pm 6.9$ & $172.7 \pm 12.4$ & $27.5 \pm 5.8$ & $10.6 \pm 2.3$ \\
\hline Cervical (LAT) & $180(91 \mathrm{M}, 89 \mathrm{~F})$ & $45.3 \pm 22.2$ & $69.7 \pm 4.7$ & $170.5 \pm 14.7$ & $23.3 \pm 2.4$ & $12 \pm 0.6$ \\
\hline Thoracic (AP) & $180(94 \mathrm{M}, 76 \mathrm{~F})$ & $37.7 \pm 17.6$ & $71.2 \pm 4.2$ & $164.6 \pm 3.3$ & $26.3 \pm 1.5$ & $20.1 \pm 2.4$ \\
\hline Thoracic (LAT) & $180(99 \mathrm{M}, 71 \mathrm{~F})$ & $44.9 \pm 17.2$ & $69.5 \pm 4.65$ & $168.8 \pm 27.2$ & $24.9 \pm 3.5$ & $32 \pm 3.1$ \\
\hline Lumbar (AP) & $180(76 \mathrm{M}, 104 \mathrm{~F})$ & $52.6 \pm 14.8$ & $72.4 \pm 3.9$ & 29.7 & $26.6 \pm 1.2$ & $20.9 \pm 1.8$ \\
\hline Lumbar (LAT) & $180(74 \mathrm{M}, 106 \mathrm{~F})$ & $53.9 \pm 13.4$ & $70.2 \pm 4.4$ & $167.2 \pm 8.1$ & $26.3 \pm 4.1$ & $33.2 \pm 2.9$ \\
\hline Chest (PA) & $180(109 \mathrm{M}, 71 \mathrm{~F})$ & $35.6 \pm 18.1$ & $68.1 \pm 5$ & $170.7 \pm 16.9$ & $25.1 \pm 1.7$ & $17.4 \pm 2.3$ \\
\hline Chest (LAT) & $180(111 \mathrm{M}, 79 \mathrm{~F})$ & $38.7 \pm 20.1$ & $70.8 \pm 6.3$ & $169.2 \pm 10.9$ & $28 \pm 3.2$ & $36 \pm 2.4$ \\
\hline Abdomen (AP) & $180(88 \mathrm{M}, 92 \mathrm{~F})$ & $54.6 \pm 17.0$ & $69.4 \pm 4.8$ & $166.1 \pm 17.8$ & $28.6 \pm 5.1$ & $20.8 \pm 1.8$ \\
\hline Pelvis (AP) & $180(105 \mathrm{M}, 75 \mathrm{~F})$ & $49.6 \pm 12.7$ & $68.9 \pm 3.3$ & $165.5 \pm 14.2$ & $29.1 \pm 2.9$ & $18.4 \pm 1.6$ \\
\hline Hip (AP) & $180(97 \mathrm{M}, 73 \mathrm{~F})$ & $54.3 \pm 10.5$ & $69 \pm 5.9$ & $166.4 \pm 15.7$ & $26.5 \pm 4.6$ & $12.1 \pm 0.9$ \\
\hline Total/Average & $2340(1237 \mathrm{M}, 1103 \mathrm{~F})$ & $46.5 \pm 17.9$ & $69.8 \pm 5.0$ & $157.0 \pm 15.5$ & $26.5 \pm 3.1$ & $19.8 \pm 1.9$ \\
\hline
\end{tabular}

Table 2. Mean exposure parameters data and ESD values for all imaging centers and examinations.

\begin{tabular}{|c|c|c|c|c|c|c|}
\hline \multirow{2}{*}{ Exam } & \multirow{2}{*}{$k V p$} & \multirow{2}{*}{ mAs } & \multirow{2}{*}{ FDD (cm) } & \multirow{2}{*}{ FSD (cm) } & \multicolumn{2}{|c|}{ ESD } \\
\hline & & & & & Mean & Max/Min \\
\hline Skull (PA) & $70.8 \pm 4.6$ & $19.2 \pm 9.8$ & $100 \pm 10.2$ & $98.4 \pm 10.5$ & $1.6 \pm 0.7$ & 7.3 \\
\hline Skull (LAT) & $70.2 \pm 5.9$ & $19.3 \pm 8.7$ & $97 \pm 8.4$ & $84.5 \pm 9.2$ & $1.8 \pm 0.9$ & 14.4 \\
\hline Cervical (AP) & $67.3 \pm 3.6$ & $17.8 \pm 5.3$ & $110 \pm 5$ & $99.4 \pm 5.3$ & $1.2 \pm 0.6$ & 10.6 \\
\hline Cervical (LAT) & $69.3 \pm 7.2$ & $18.6 \pm 3.9$ & $110 \pm 4.3$ & $98 \pm 4.7$ & $1.2 \pm 0.6$ & 7.2 \\
\hline Thoracic (AP) & $73.9 \pm 4.4$ & $27.6 \pm 7.2$ & $107 \pm 4.6$ & $86.9 \pm 3.8$ & $2.8 \pm 1.2$ & 11.7 \\
\hline Thoracic (LAT) & $79.8 \pm 4.8$ & $42.4 \pm 10.1$ & $109 \pm 5.0$ & $77 \pm 5.0$ & $8.3 \pm 2.8$ & 4.9 \\
\hline Lumbar (AP) & $77 \pm 5.2$ & $29.7 \pm 5.8$ & $113 \pm 5.1$ & $92.1 \pm 5.3$ & $4.2 \pm 2.5$ & 15.8 \\
\hline Lumbar (LAT) & $85.2 \pm 5.7$ & $44.5 \pm 7.7$ & $114 \pm 3.8$ & $80.8 \pm 4.1$ & $9.4 \pm 4.0$ & 9.9 \\
\hline Chest (PA) & $73.2 \pm 4.5$ & $22.3 \pm 9.8$ & $150 \pm 8.9$ & $132.6 \pm 8.3$ & $1 \pm 0.6$ & 14.9 \\
\hline Chest (LAT) & $84.3 \pm 3.9$ & $35.5 \pm 6.1$ & $135 \pm 7.1$ & $99 \pm 6.5$ & $1.7 \pm 0.8$ & 14.4 \\
\hline Abdomen (AP) & $76.3 \pm 5.2$ & $28.3 \pm 8.1$ & $85 \pm 5.2$ & $64.2 \pm 5.3$ & $3.3 \pm 1.9$ & 41 \\
\hline Pelvis (AP) & $72.2 \pm 6.6$ & $22.2 \pm 3.9$ & $80 \pm 4.9$ & $61.6 \pm 4.0$ & $2.1 \pm 1.3$ & 10.2 \\
\hline Hip (AP) & $68.8 \pm 4.9$ & $20.2 \pm 2.2$ & $80 \pm 4.8$ & $67.9 \pm 4.9$ & $1.6 \pm 0.7$ & 8.7 \\
\hline Average & $74.5 \pm 5.1$ & $26.7 \pm 6.8$ & $106.9 \pm 5.9$ & $87.9 \pm 1.4$ & $3.1 \pm 1.4$ & $13.2 \pm 9.0$ \\
\hline
\end{tabular}

$\mathrm{AP}$, anterior-posterior projection; PA, posterior-anterior projection; LAT, lateral projection 


\section{Entrance surface air kerma (ESAK, mGy) and ESD calculation}

Patient data were obtained from recorded documents. Furthermore, radiation parameters such as $\mathrm{kVp}$, mAs, focal area to detector distance (FDD, $\mathrm{cm}$ ), and focal area to skin distance (FSD, $\mathrm{cm}$ ) were measured.

A calibrated semiconductor dosimeter (Baracuda, RTI Electronics, Sweden) was used for measuring air kerma (mGy) at the energy level of $40-150 \mathrm{kVp}$, in $10 \mathrm{kVp}$ steps. In addition, the distance from X-ray tube and field size were chosen at standard conditions $(100$ $\mathrm{cm}$ and $10 \times 10 \mathrm{~cm}^{2}$, respectively). In equation 1 , the obtaining method of entrance surface air kerma (ESAK, mGy) is expressed (4):

$\mathrm{ESAK}=\mathrm{D}_{\text {air }} \times(\mathrm{FDD} / \mathrm{FSD})^{2} \times \mathrm{BSF}$

Where $D_{\text {air }}$ is the air dose obtained by the dosimeter in air (mGy). BSF (has no unit) refers to backscatter factor and it was between 1.3-1.5 according to the IAEA technical report series no. 457 (33).

As described in IAEA recommendation (33), ESD was obtained by equation 2 , where 1.06 is the ratio of mass-energy absorption coefficients for tissue and air.

$\mathrm{ESD}=\mathrm{ESAK} \times 1.06$

\section{Calculation of mean organ dose, induced cancer risks and mortalities}

Mean organ doses were calculated using PCXMC 2.0 software (STUK-Radiation and Nuclear Authority, Helsinki, Finland) based on the ESD values and radiation parameters such as KVp, mAs, FDD, filter thickness, and field size.

The calculated mean organ dose for each digital radiology exam was used to estimate the cancer risk and mortality for irradiated regions using the mean annual number of examinations in Mazandaran province. Following the previous study, there have been approximately 662402 radiographies performed per year and the total population is 2976219 showing that the percentage ratio of radiography exams to the whole population is $22.26 \%$ (34).

Various types of cancer risks and mortalities have been evaluating by BEIR VII-Phase 2 model (20). Briefly, in this model, a low dose limit, doses less than 100 mGy and a gradual dose limit of 0.1 $\mathrm{mGy} / \mathrm{min}$ are defined. Moderating factors are considered for cancer type, gender, age at exposure, and time elapsed after exposure (19). In this model, for estimating solid tumors and the risk of leukemia, a threshold-free linear model and quadratic linear model were used. The dose and dose rate effectiveness factor of 1.5 was used to convert the risk in high doses (rates) to the risk in low doses (rates). In other words, the risk in the high dose is divided by 1.5 to reach the risk in the low dose. The report uses an exponential multiple-risk estimation model of the natural risk frequency in the community. A combination of progressive and incremental models have been used for estimating the cancer risk based on the age at radiation time (between progressive and incremental models), and also in some cancers such as thyroid, the progressive model was applied. For some other cancers like breast cancer in women, the incremental model and the weighted mean of both methods were used. In the expression of risk, the committee has finally presented the life attributed risk (20).

\section{Frequency contribution in cancer risk estimation}

The risks of cancer incidence and mortalities in the whole population for each of the radiology exams were calculated through multiplying the calculated risks by the frequency of the examination and the percentage ratio of radiography exams to the whole population. The total risks from all examinations were evaluated by summation of all the risks.

\section{Statistical analysis}

The assumption of normality of data distribution was checked by Kolmogorov-Smirnov (K-S) test. The correlations between the age and lifetime cancer induction and mortality risks were evaluated using Pearson's correlation, and $\mathrm{R}^{2}$ values were considered as an index of correlation power. Furthermore, the differences 
of lifetime cancer induction and mortality risks between men and women were assessed by independent T-tests in various age groups. The level of statistical significance was set at $\mathrm{P}<0.05$. All of the statistical analyses were performed in SPSS software version 19 (Chicago, Illinois, US).

\section{RESULTS}

\section{ESD values and mean organ dose}

The ESD values for each digital radiography examination are shown in table 2 . According to the table, these values range from 4.9 to 41 for the lateral thoracic spine and the abdomen, respectively. This variation can be explained with regard to some reasons such as different types of radiography systems, selection of exposure parameters, and the distance of tube to the patient.

Mean organ dose (brain, lung, liver, stomach, colon, bladder, breast, prostate, ovary, and uterus) values for each of 13 selected radiography exams are shown in table 3 . The effective dose and cancer risk are dependent on the kind of examination, for example, during

Table 3. Mean effective doses (mSv) at various organs in all digital radiography imaging techniques

\begin{tabular}{|c|c|c|c|c|c|c|c|c|c|c|}
\hline Examination & Brain & Lung & Liver & Stomach & Colon & Bladder & Breast & Prostate & Ovary & Uterus \\
\hline Skull (PA) & $0.16 \pm 0.02$ & $0.0 \pm 0.0$ & $0.0 \pm 0.0$ & $0.0 \pm 0.0$ & $0.0 \pm 0.0$ & $0.0 \pm 0.0$ & $0.0 \pm 0.0$ & $0.0 \pm 0.0$ & $0.0 \pm 0.0$ & $0.0 \pm 0.0$ \\
\hline Skull (LAT) & $0.18 \pm 0.04$ & $0.0 \pm 0.0$ & $0.0 \pm 0.0$ & $0.0 \pm 0.0$ & $0.0 \pm 0.0$ & $0.0 \pm 0.0$ & $0.0 \pm 0.0$ & $0.0 \pm 0.0$ & $0.0 \pm 0.0$ & $0.0 \pm 0.0$ \\
\hline $\begin{array}{c}\text { Cervical spine } \\
\text { (AP) }\end{array}$ & $0.08 \pm 0.02$ & $0.14 \pm 0.04$ & $0.05 \pm 0.01$ & $0.03 \pm 0.01$ & $0.01 \pm 0.0$ & $0.0 \pm 0.0$ & $0.02 \pm 0.01$ & $0.0 \pm 0.0$ & $0.0 \pm 0.0$ & $0.0 \pm 0.0$ \\
\hline $\begin{array}{c}\text { Cervical spine } \\
\text { (LAT) }\end{array}$ & $0.05 \pm 0.01$ & $0.10 \pm 0.02$ & $0.03 \pm 0.01$ & $0.01 \pm 0.0$ & $0.0 \pm 0.0$ & $0.0 \pm 0.0$ & $0.06 \pm 0.02$ & $0.0 \pm 0.0$ & $0.0 \pm 0.0$ & $0.0 \pm 0.0$ \\
\hline $\begin{array}{c}\text { Thoracic spine } \\
\text { (AP) }\end{array}$ & $0.01 \pm 0.0$ & $0.14 \pm 0.04$ & $0.21 \pm 0.05$ & $0.26 \pm 0.05$ & $0.04 \pm 0.01$ & $0.00 \pm 0.0$ & $0.26 \pm 0.06$ & $0.00 \pm 0.0$ & $0.02 \pm 0.0$ & $0.02 \pm 0.0$ \\
\hline $\begin{array}{c}\text { Thoracic spine } \\
\text { (LAT) }\end{array}$ & $0.01 \pm 0.0$ & $0.11 \pm 0.2$ & $0.24 \pm 0.4$ & $0.26 \pm 0.04$ & $0.03 \pm 0.0$ & $0.00 \pm 0.0$ & $0.17 \pm 0.03$ & $0.00 \pm 0.0$ & $0.02 \pm 0.0$ & $0.01 \pm 0.0$ \\
\hline $\begin{array}{c}\text { Lumbar spine } \\
\text { (AP) }\end{array}$ & $0.0 \pm 0.0$ & $0.08 \pm 0.02$ & $0.27 \pm 0.07$ & $0.21 \pm 0.05$ & $0.06 \pm 0.01$ & $0.03 \pm 0.01$ & $0.08 \pm 0.02$ & $0.07 \pm 0.02$ & $0.21 \pm 0.04$ & $0.25 \pm 0.05$ \\
\hline $\begin{array}{c}\text { Lumbar spine } \\
\text { (LAT) }\end{array}$ & $0.0 \pm 0.0$ & $0.06 \pm 0.01$ & $0.33 \pm 0.08$ & $0.29 \pm 0.07$ & $0.19 \pm 0.05$ & $0.04 \pm 0.01$ & $0.01 \pm 0.0$ & $0.05 \pm 0.01$ & $0.16 \pm 0.04$ & $0.18 \pm 0.04$ \\
\hline Chest (PA) & $0.01 \pm 0.0$ & $0.18 \pm 0.05$ & $0.14 \pm 0.05$ & $0.13 \pm 0.03$ & $0.01 \pm 0.0$ & $0.02 \pm 0.0$ & $0.11 \pm 0.02$ & $0.01 \pm 0.0$ & $0.0 \pm 0.0$ & $0.00 \pm 0.0$ \\
\hline Chest (LAT) & $0.0 \pm 0.0$ & $0.21 \pm 0.04$ & $0.15 \pm 0.03$ & $0.25 \pm 0.06$ & $0.02 \pm 0.01$ & $0.01 \pm 0.0$ & $0.24 \pm 0.06$ & $0.00 \pm 0.0$ & $0.00 \pm 0.0$ & $0.00 \pm 0.0$ \\
\hline Abdomen (AP) & $0.0 \pm 0.0$ & $0.11 \pm 0.02$ & $0.48 \pm 0.09$ & $0.23 \pm 0.05$ & $0.81 \pm 0.13$ & $0.28 \pm 0.05$ & $0.36 \pm 0.07$ & $0.22 \pm 0.05$ & $0.47 \pm 0.09$ & $0.51 \pm 0.11$ \\
\hline Pelvis (AP) & $0.0 \pm 0.0$ & $0.08 \pm 0.02$ & $0.06 \pm 0.02$ & $0.14 \pm 0.05$ & $0.95 \pm 0.15$ & $1.16 \pm 0.22$ & $0.03 \pm 0.0$ & $0.71 \pm 0.11$ & $0.88 \pm 0.15$ & $0.92 \pm 0.18$ \\
\hline Hip (AP) & $0.0 \pm 0.0$ & $0.00 \pm 0.0$ & $0.00 \pm 0.0$ & $0.01 \pm 0.0$ & $0.19 \pm 0.4$ & $0.39 \pm 0.7$ & $0.0 \pm 0.0$ & $0.83 \pm 0.12$ & $0.17 \pm 0.03$ & $0.26 \pm 0.06$ \\
\hline Average & $0.04 \pm 0.01$ & $0.09 \pm 0.02$ & $0.15 \pm 0.05$ & $0.14 \pm 0.03$ & $0.18 \pm 0.04$ & $0.15 \pm 0.03$ & $0.10 \pm 0.02$ & $0.15 \pm 0.03$ & $0.15 \pm 0.02$ & $0.16 \pm 0.03$ \\
\hline
\end{tabular}

skull examination, the brain has a higher risk compared to the others.

\section{Frequency contribution}

Figure 1 illustrates the percentage of frequency contributions for each examination. According to this figure, chest examination (PA and LAT) is the largest contributor $(50.2 \%)$. Therefore, it is expected that the organs involved in this examination (like breast and lung) have a higher cancer risk.

\section{Cancer risks and mortalities}

In tables 4 and 5, the lifetime attributable risk of cancer incidence and mortality induced by a one year exposure to digital radiography examinations (in 100,000 people) for various sites of cancers at different ages for both genders have been represented. Also, in these tables, the total of additional risks of all cancers for ages 


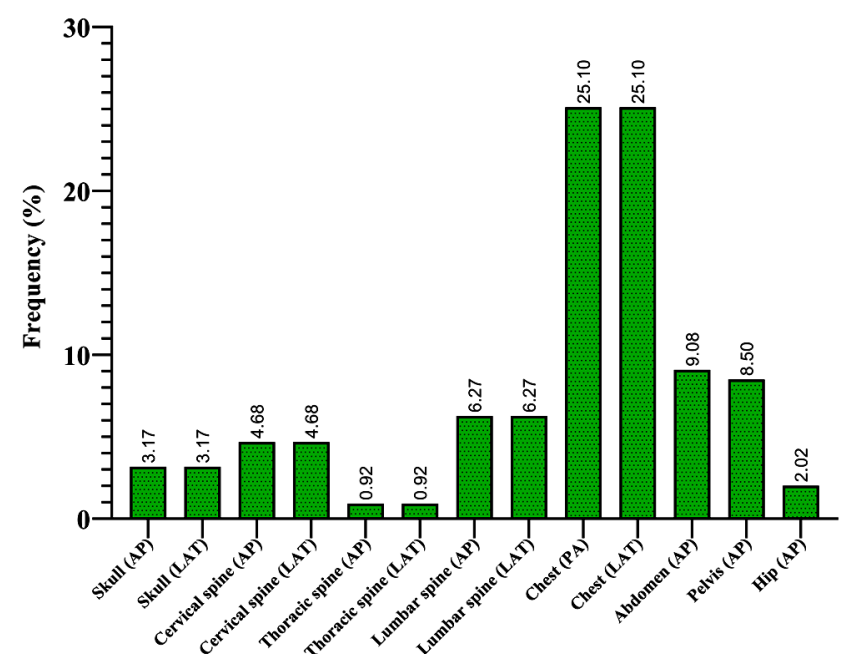

Figure 1. Distribution of the frequency (\%) for the examinations conducted in Mazandaran province.

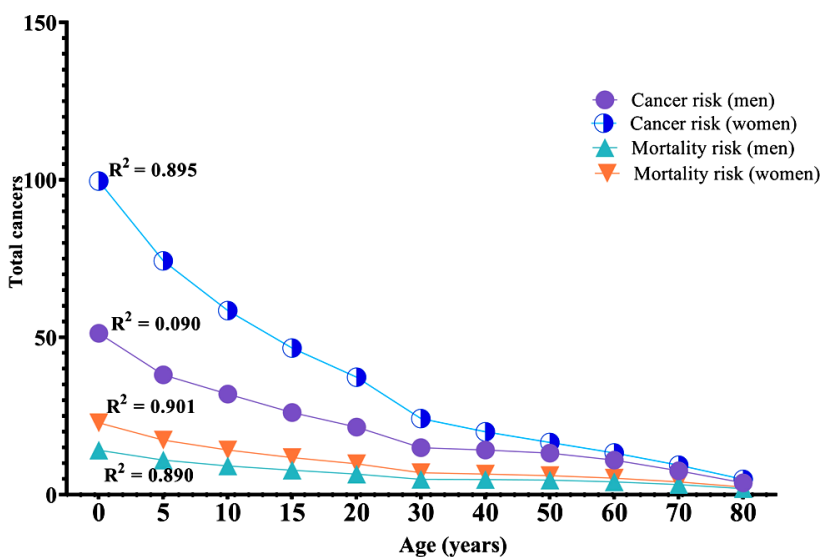

Figure 2. Comparison between men and women for all cancer risks and mortalities induced by radiations from radiography examinations for one year (incidence probability in 100,000 people) regarding the ages. Also $\mathrm{R}^{2}$ values were indicate for each curve.

Table 4. Mean ( \pm SD) values of lifetime risk of various cancers incidence (in 100,000 people) induced by radiations from radiography examinations for one year in Mazandaran province.

\begin{tabular}{|c|c|c|c|c|c|c|c|c|c|c|c|}
\hline \multicolumn{12}{|c|}{ Age at exposure time (year) } \\
\hline & 0 & 5 & 10 & 15 & 20 & 30 & 40 & 50 & 60 & 70 & 80 \\
\hline \multicolumn{12}{|l|}{ Male } \\
\hline Stomach & $2.60 \pm 0.54$ & $2.22 \pm 0.52$ & $1.87 \pm 0.47$ & $1.56 \pm 0.42$ & $1.35 \pm 0.51$ & $0.95 \pm 0.33$ & $0.92 \pm 0.25$ & $0.88 \pm 0.23$ & $0.71 \pm 0.19$ & $0.44 \pm 0.11$ & $0.22 \pm 0.05$ \\
\hline Colon & $6.31 \pm 0.74$ & $5.35 \pm 0.72$ & $4.52 \pm 0.68$ & $3.77 \pm 0.62$ & $3.20 \pm 0.63$ & $2.31 \pm 0.48$ & $2.25 \pm 0.51$ & $2.08 \pm 0.42$ & $1.73 \pm 0.35$ & $1.19 \pm 0.24$ & $0.55 \pm 0.10$ \\
\hline Liver & $1.56 \pm 0.31$ & $1.30 \pm 0.19$ & $1.12 \pm 0.17$ & $0.93 \pm 0.11$ & $0.77 \pm 0.09$ & $0.56 \pm 0.07$ & $0.53 \pm 0.06$ & $0.48 \pm 0.05$ & $0.35 \pm 0.05$ & $0.20 \pm 0.04$ & $0.07 \pm 0.01$ \\
\hline Lung & $12.42 \pm 1.15$ & $10.32 \pm 1.03$ & $8.54 \pm 0.94$ & $7.12 \pm 0.83$ & $5.89 \pm 0.71$ & $4.15 \pm 0.65$ & $4.13 \pm 0.62$ & $4.09 \pm 0.54$ & $3.60 \pm 0.39$ & $2.63 \pm 0.34$ & $1.37 \pm 0.31$ \\
\hline Prostate & $2.13 \pm 0.48$ & $1.83 \pm 0.42$ & $1.53 \pm 0.33$ & $1.30 \pm 0.30$ & $1.09 \pm 0.28$ & $0.79 \pm 0.22$ & $0.79 \pm 0.23$ & $0.75 \pm 0.17$ & $0.59 \pm 0.12$ & $0.32 \pm 0.08$ & $0.11 \pm 0.03$ \\
\hline Bladder & $4.15 \pm 0.57$ & $3.51 \pm 0.48$ & $2.97 \pm 0.45$ & $2.51 \pm 0.39$ & $2.13 \pm 0.37$ & $1.56 \pm 0.29$ & $1.56 \pm 0.21$ & $1.51 \pm 0.24$ & $1.31 \pm 0.18$ & $0.93 \pm 0.15$ & $0.45 \pm 0.08$ \\
\hline Other & $15.67 \pm 1.43$ & $9.38 \pm 1.05$ & $8.41 \pm 0.91$ & $6.59 \pm 0.74$ & $5.21 \pm 0.65$ & $3.31 \pm 0.48$ & $2.87 \pm 0.41$ & $2.33 \pm 0.37$ & $1.63 \pm 0.31$ & $0.95 \pm 0.24$ & $0.38 \pm 0.09$ \\
\hline \begin{tabular}{|l|} 
Thyroid \\
\end{tabular} & $3.39 \pm 0.44$ & $2.24 \pm 0.37$ & $1.47 \pm 0.21$ & $0.97 \pm 0.14$ & $0.62 \pm 0.07$ & $0.27 \pm 0.04$ & $0.09 \pm 0.02$ & $0.03 \pm 0.01$ & $0.01 \pm 0.00$ & $0.00 \pm 0.00$ & $0.00 \pm 0.00$ \\
\hline All solids & $48.23 \pm 4.12$ & $36.15 \pm 3.81$ & $30.43 \pm 3.24$ & $24.75 \pm 2.54$ & $20.26 \pm 2.10$ & $13.90 \pm 1.26$ & $13.14 \pm 1.35$ & $12.15 \pm 1.26$ & $9.93 \pm 1.06$ & $6.66 \pm 0.65$ & $3.15 \pm 0.51$ \\
\hline Leukemia & $3.06 \pm 0.41$ & $1.92 \pm 0.25$ & $1.55 \pm 0.22$ & $1.35 \pm 0.18$ & $1.24 \pm 0.16$ & $1.08 \pm 0.13$ & $1.08 \pm 0.08$ & $1.08 \pm 0.07$ & $1.06 \pm 0.07$ & $0.94 \pm 0.06$ & $0.62 \pm 0.06$ \\
\hline \multicolumn{12}{|l|}{ Female } \\
\hline Stomach & $3.45 \pm 0.47$ & $2.90 \pm 0.38$ & $2.45 \pm 0.33$ & $2.08 \pm 0.29$ & $1.77 \pm 0.25$ & $1.22 \pm 0.42$ & $1.19 \pm 0.34$ & $1.11 \pm 0.32$ & $0.94 \pm 0.22$ & $0.66 \pm 0.13$ & $0.38 \pm 0.5$ \\
\hline Colon & $4.13 \pm 0.52$ & $3.51 \pm 0.41$ & $2.97 \pm 0.38$ & $2.52 \pm 0.33$ & $2.14 \pm 0.28$ & $1.54 \pm 0.21$ & $1.48 \pm 0.19$ & $1.37 \pm 0.18$ & $1.27 \pm 0.17$ & $1.08 \pm 0.12$ & $0.55 \pm 0.08$ \\
\hline Liver & $0.72 \pm 0.12$ & $0.59 \pm 0.07$ & $0.51 \pm 0.07$ & $0.41 \pm 0.06$ & $0.36 \pm 0.05$ & $0.26 \pm 0.05$ & $0.26 \pm 0.05$ & $0.23 \pm 0.04$ & $0.18 \pm 0.03$ & $0.13 \pm 0.03$ & $0.05 \pm 0.01$ \\
\hline Lung & $21.99 \pm 2.41$ & $18.24 \pm 2.12$ & $15.12 \pm 1.94$ & $12.51 \pm 1.47$ & $10.38 \pm 1.26$ & $7.26 \pm 0.91$ & $7.23 \pm 0.88$ & $6.93 \pm 0.82$ & $6.06 \pm 0.75$ & $4.43 \pm 0.58$ & $2.32 \pm 0.37$ \\
\hline Breast & $33.94 \pm 3.36$ & $26.49 \pm 2.55$ & $20.63 \pm 2.44$ & $16.02 \pm 2.31$ & $12.43 \pm 1.67$ & $7.33 \pm 0.94$ & $4.08 \pm 0.74$ & $2.02 \pm 0.41$ & $0.89 \pm 0.18$ & $0.34 \pm 0.05$ & $0.11 \pm 0.03$ \\
\hline Uterus & $1.17 \pm 0.24$ & $0.98 \pm 0.18$ & $0.84 \pm 0.18$ & $0.70 \pm 0.14$ & $0.61 \pm 0.08$ & $0.42 \pm 0.07$ & $0.37 \pm 0.05$ & $0.30 \pm 0.04$ & $0.21 \pm 0.03$ & $0.12 \pm 0.02$ & $0.05 \pm 0.01$ \\
\hline Ovary & $2.11 \pm 0.35$ & $1.76 \pm 0.28$ & $1.48 \pm 0.21$ & $1.22 \pm 0.23$ & $1.02 \pm 0.16$ & $0.69 \pm 0.13$ & $0.63 \pm 0.08$ & $0.51 \pm 0.07$ & $0.37 \pm 0.06$ & 0.23 & $0.10 \pm 0.02$ \\
\hline Bladder & $4.23 \pm 0.64$ & $3.59 \pm 0.48$ & $3.03 \pm 0.41$ & $2.57 \pm 0.37$ & $2.17 \pm 0.32$ & $1.57 \pm 0.24$ & $1.56 \pm 0.21$ & $1.48 \pm 0.19$ & $1.28 \pm 0.18$ & $0.94 \pm$ & $0.47 \pm 0.09$ \\
\hline Other & $16.38 \pm 1.83$ & $8.79 \pm 1.22$ & $6.39 \pm 1.54$ & $5.00 \pm 0.74$ & $3.95 \pm 0.51$ & $2.53 \pm 0.37$ & $2.21 \pm 0.31$ & $1.81 \pm 0.24$ & $1.33 \pm 0.19$ & $0.83 \pm 0.15$ & $0.37 \pm 0.07$ \\
\hline Thyroid & $9.11 \pm 1.36$ & $6.02 \pm 1.07$ & $3.95 \pm 0.71$ & $2.56 \pm 0.55$ & $1.62 \pm 0.30$ & $0.59 \pm 0.08$ & $0.20 \pm 0.05$ & $0.06 \pm 0.01$ & $0.01 \pm 0.00$ & $0.00 \pm 0.00$ & $0.00 \pm 0.00$ \\
\hline All solids & $97.23 \pm 7.28$ & $72.87 \pm 6.63$ & $57.37 \pm 5.28$ & $45.59 \pm 4.79$ & $36.45 \pm 3.28$ & $23.41 \pm 2.44$ & $19.21 \pm 2.12$ & $15.82 \pm 1.75$ & $12.54 \pm 1.36$ & $8.76 \pm 1.07$ & $4.40 \pm 0.52$ \\
\hline Leukemia & $2.39 \pm 0.31$ & $1.44 \pm 0.20$ & $1.11 \pm 0.17$ & $0.98 \pm 0.12$ & $0.92 \pm 0.10$ & $0.81 \pm 0.09$ & $0.80 \pm 0.08$ & $0.80 \pm 0.08$ & $0.74 \pm 0.08$ & $0.66 \pm 0.06$ & $0.48 \pm 0.05$ \\
\hline
\end{tabular}


Table 5. Mean $( \pm S D$ ) values of lifetime risk of various cancers mortality (in 100,000 people) induced by radiations from radiography examinations for one year in Mazandaran province.

\begin{tabular}{|c|c|c|c|c|c|c|c|c|c|c|c|}
\hline \multicolumn{12}{|c|}{ Age at exposure time (year) } \\
\hline & $\mathbf{0}$ & 5 & 10 & 15 & 20 & 30 & 40 & 50 & 60 & 70 & 80 \\
\hline \multicolumn{12}{|l|}{ Male } \\
\hline Stomach & $0.53 \pm 0.07$ & $0.44 \pm 0.05$ & $0.39 \pm 0.05$ & $0.32 \pm 0.04$ & $0.27 \pm 0.04$ & $0.21 \pm 0.03$ & \begin{tabular}{|l|l|}
$30.19 \pm 0.03$ \\
\end{tabular} & $0.17 \pm 0.02$ & $0.14 \pm 0.02$ & $0.10 \pm 0.01$ & $0.05 \pm 0.01$ \\
\hline Colon & $2.10 \pm 0.34$ & $1.79 \pm 0.25$ & $1.51 \pm 0.21$ & $1.28 \pm 0.18$ & $1.08 \pm 0.13$ & $0.79 \pm 0.11$ & $0.77 \pm 0.08$ & $0.74 \pm 0.07$ & $0.63 \pm 0.07$ & $0.46 \pm 0.06$ & $0.27 \pm 0.03$ \\
\hline Liver & $0.57 \pm 0.07$ & $0.48 \pm 0.06$ & $0.40 \pm 0.06$ & $0.35 \pm 0.05$ & $0.30 \pm 0.04$ & $0.21 \pm 0.04$ & \begin{tabular}{l|l|}
$40.21 \pm 0.04$ \\
\end{tabular} & $0.18 \pm 0.03$ & $0.15 \pm 0.03$ & $0.10 \pm 0.02$ & $0.05 \pm 0.01$ \\
\hline Lung & $4.10 \pm 0.53$ & $3.41 \pm 0.47$ & $2.83 \pm 0.35$ & $2.35 \pm 0.32$ & $1.95 \pm 0.27$ & $1.38 \pm 0.22$ & $21.38 \pm 0.22$ & $1.34 \pm 0.18$ & $1.20 \pm 0.15$ & $0.92 \pm 0.13$ & $0.54 \pm 0.07$ \\
\hline Prostate & $0.22 \pm 0.04$ & $0.19 \pm 0.04$ & $0.15 \pm 0.03$ & $0.13 \pm 0.03$ & 0.12 & 0.0 & \pm 0.02 & $0.09 \pm 0.02$ & $0.09 \pm 0.02$ & & $0.06 \pm 0.01$ \\
\hline Bladder & $0.58 \pm 0.07$ & $0.49 \pm 0.07$ & $0.41 \pm 0.06$ & $0.35 \pm 0.06$ & $0.30 \pm 0.05$ & $0.22 \pm 0.04$ & \begin{tabular}{ll|}
$40.22 \pm 0.04$ \\
\end{tabular} & $0.22 \pm 0.04$ & $0.22 \pm 0.04$ & $0.19 \pm 0.03$ & $0.13 \pm 0.02$ \\
\hline Other & $5.16 \pm 0.62$ & $3.29 \pm 0.38$ & $2.58 \pm 0.31$ & $2.09 \pm 0.27$ & $1.73 \pm 0.22$ & 1.21 & $1.14 \pm 0.15$ & $0.99 \pm 0.12$ & $0.75 \pm 0.08$ & .06 & $0.22 \pm 0.04$ \\
\hline All solids & $13.26 \pm 1.53$ & $10.07 \pm 1.26$ & $8.27 \pm 1.11$ & $6.88 \pm 0.92$ & $5.73 \pm 0.83$ & $4.09 \pm 0.61$ & $14.00 \pm 0.59$ & $3.73 \pm 0.47$ & $3.17 \pm 0.45$ & .36 & \begin{tabular}{l|l}
$5.32 \pm 0.21$ \\
\end{tabular} \\
\hline Leukemia & $0.92 \pm 0.09$ & \begin{tabular}{|l|}
$0.92 \pm 0.09$ \\
\end{tabular} & $0.92 \pm 0.09$ & $0.90 \pm 0.09$ & $0.86 \pm 0.08$ & $0.83 \pm 0.08$ & $8.86 \pm 0.09$ & $0.92 \pm 0.11$ & $0.94 \pm 0.12$ & $0.89 \pm 0.09$ & $0.66 \pm 0.07$ \\
\hline \multicolumn{12}{|l|}{\begin{tabular}{|l|} 
Female \\
\end{tabular}} \\
\hline Stomach & $0.74 \pm 0.07$ & $0.62 \pm 0.07$ & $0.53 \pm 0.06$ & $0.44 \pm 0.05$ & \begin{tabular}{|l|}
$0.37 \pm 0.04$ \\
\end{tabular} & $0.27 \pm 0.04$ & $0.26 \pm 0.03$ & $0.25 \pm 0.03$ & $0.21 \pm 0.03$ & $0.17 \pm 0.02$ & $0.10 \pm 0.02$ \\
\hline Colon & $1.32 \pm 0.15$ & $1.11 \pm 0.13$ & $0.94 \pm 0.11$ & $0.80 \pm 0.09$ & $0.68 \pm 0.08$ & $0.49 \pm 0.07$ & $0.48 \pm 0.07$ & $0.45 \pm 0.06$ & $0.40 \pm 0.05$ & $0.32 \pm 0.05$ & $0.19 \pm 0.03$ \\
\hline Liver & $0.31 \pm 0.04$ & $0.26 \pm 0.04$ & $0.22 \pm 0.03$ & $0.18 \pm 0.03$ & $0.15 \pm 0.02$ & $0.12 \pm 0.02$ & $0.10 \pm 0.02$ & $0.10 \pm 0.01$ & $0.09 \pm 0.01$ & $0.06 \pm 0.01$ & $0.04 \pm 0.01$ \\
\hline Lung & $8.29 \pm 0.91$ & $6.89 \pm 0.85$ & $5.70 \pm 0.74$ & $4.73 \pm 0.62$ & $3.93 \pm 0.51$ & $2.75 \pm 0.38$ & $2.73 \pm 0.32$ & $2.63 \pm 0.32$ & $2.36 \pm 0.29$ & $1.81 \pm 0.27$ & $1.04 \pm 0.17$ \\
\hline Breast & $3.53 \pm 0.46$ & $2.76 \pm 0.39$ & $2.15 \pm 0.31$ & $1.68 \pm 0.26$ & $1.30 \pm 0.20$ & 0.79 & 0.4 & $0.25 \pm 0.07$ & $0.12 \pm 0.04$ & \pm 0.02 & $0.03 \pm 0.01$ \\
\hline Uterus & $0.14 \pm 0.02$ & $0.13 \pm 0.02$ & $0.10 \pm 0.02$ & $0.09 \pm 0.01$ & $0.08 \pm 0.01$ & $0.05 \pm 0.01$ & $0.05 \pm 0.01$ & $0.04 \pm 0.01$ & $0.04 \pm 0.01$ & $0.03 \pm 0.01$ & $0.01 \pm 0.00$ \\
\hline Ovary & $0.71 \pm 0.07$ & $0.64 \pm 0.07$ & $0.50 \pm 0.07$ & $0.44 \pm 0.05$ & $0.36 \pm 0.05$ & $0.26 \pm 0.04$ & $0.26 \pm 0.04$ & $0.23 \pm 0.03$ & $0.19 \pm 0.03$ & $0.13 \pm 0.02$ & $0.06 \pm 0.02$ \\
\hline Bladder & $0.76 \pm 0.09$ & $0.66 \pm 0.08$ & $0.55 \pm 0.07$ & $0.46 \pm 0.06$ & $0.40 \pm 0.06$ & $0.30 \pm 0.05$ & $0.30 \pm 0.05$ & $0.28 \pm 0.04$ & $0.28 \pm 0.04$ & $0.25 \pm 0.03$ & \begin{tabular}{|l|l}
$30.17 \pm 0$. \\
\end{tabular} \\
\hline Other & $6.33 \pm 0.81$ & $3.70 \pm 0.52$ & $2.84 \pm 0.47$ & $2.31 \pm 0.36$ & $1.90 \pm 0.27$ & $1.33 \pm 0.21$ & $1.25 \pm 0.20$ & $1.11 \pm 0.18$ & $0.89 \pm 0.15$ & $0.61 \pm 0.11$ & $0.31 \pm 0$. \\
\hline All solids & $22.15 \pm 2.52$ & $16.71 \pm 1.88$ & $13.56 \pm 1.61$ & $11.12 \pm 1.32$ & $9.17 \pm 1.05$ & $6.33 \pm 0.84$ & $5.87 \pm 0.76$ & $5.35 \pm 0.64$ & $4.57 \pm 0.56$ & $3.42 \pm 0.50$ & $1.96 \pm 0.27$ \\
\hline Leukemia & $0.68 \pm 0.07$ & $0.67 \pm 0.07$ & $0.68 \pm 0.07$ & $0.67 \pm 0.07$ & $0.66 \pm 0.07$ & $0.66 \pm 0.07$ & $0.67 \pm 0.07$ & $0.70 \pm 0.07$ & $0.71 \pm 0.08$ & $0.67 \pm 0.07$ & $0.49 \pm 0.06$ \\
\hline
\end{tabular}

ranging from 0 to 80 years for men and women is depicted.

According to figure 2 , the average $( \pm S D)$ cancer risks from radiography examinations are $51.29 \pm 4.73$ and $99.62 \pm 7.36$ for new-born males and females, respectively. This risk decreases and goes down to $3.77 \pm 0.62$ and $4.88 \pm 0.07$ for men and women at the age of 80 years, in that order. The average $( \pm$ SD) mortality risks were obtained at $14.18 \pm 1.62$ and $22.83 \pm 2.55$ for new-born males and females, respectively. This risk reduced with age and fell $1.97 \pm 0.27$ and $2.45 \pm 0.38$ for men and women at the age of 80 years, respectively.

The correlation values between the age and lifetime cancer induction and mortality risks are illustrated in Figure 2. All of the risks in men and women had very strong inverse correlations with age $\left(\mathrm{R}^{2}>0.89\right)$.

Men and women of up to 50 years of age groups showed significant differences in the induced lifetime cancer risks $(\mathrm{P}<0.02)$. Furthermore, there were significant differences between men and women in lifetime cancer mortality risk up to 15 years of age group $(\mathrm{P}<0.05)$.

\section{DISCUSSION}

In this study, the cancer incident risks and mortalities induced by one of year digital radiography examinations were assessed for patients of different ages, and genders. The risks were calculated based on the BEIR VII-Phase 2 model to assess health risks from exposure to low levels of ionization radiation (20).

There are several reports, studying the patients' dose and cancer risk from diagnostic imaging procedures to obtain the DRLs and health risks of radiological imaging (13-27) based on ESD values. In a study by Khoshdel-Navi et al. (24), researchers tried to introduce DRLs for conventional radiography examinations in Mazandaran province. In their study, mean ESD values (in mGy) have been obtained at $1.47 \pm 0.98$ for skull (PA/AP), $1.01 \pm 0.79$ for skull (LAT), $0.67 \pm 0.38$ for cervical spine (AP), $0.79 \pm 0.37$ for cervical (LAT), $0.49 \pm 0.38$ for chest (PA/AP), $1.06 \pm 0.44$ for chest (LAT), $2.15 \pm 0.73$ 
for thoracic spine (AP), $3 \pm 0.87$ for thoracic spine (LAT), $2.81 \pm 0.82$ for lumbar spine (AP), $4.28 \pm 0.78$ for lumbar (LAT), $2.07 \pm 1.17$ for abdomen and $1.90 \pm 0.99$ for pelvis, respectively. Their obtained values were greater than our ESD results for almost all examinations except chest imaging (AP/PA and LAT). The higher values in Khoshdel-Navi results (24) are due to higher applied exposure parameters. In addition, in our study the exposure parameters in chest radiographies were higher, therefore, they are expected to have a higher ESDs. In our study, the ESD values for chest examination in both projections, PA and LAT, were higher than those of recommended by the UK (35), Brazil (36) and Slovenia (37).

Owning to the results, women have a higher cancer probability risk in comparison to men. This may be due to the higher cancer risk of the breast in females compared to the negligible incidence of prostate cancer risk in males. Furthermore, based on the BEIR VII-phase2 report some of the cancers like stomach, lung, and thyroid have higher incidence risk in women compared to men with the same irradiation (20).

Law et al. (38), calculated the lifetime attributed cancer risk for both genders at the ages of 5 and 30 years. The patients were exposed to digital radiography system for spine examination (PA and LAT). They have found that the cumulative lifetime attributable cancer risk ranges from 0.08 to $0.17 \%$ in Asian and Western populations. In the present study, the total lifetime cancer risk for 5 and 30 years age groups was estimated at $0.38 \pm 0.37$ and $0.15 \pm$ $0.02 \%$ for men, and $0.74 \pm 0.07$ and $0.24 \pm$ $0.03 \%$ for women, respectively. These values are approximately 2.5 times higher than Law et al. study which can be explained by the different in radiography systems, radiation parameters, and cancer risk estimation models.

Mazonakis et al. (39) investigated the lifetime mortality risks for children (four groups) during skull radiographies in AP, PA, and LAT positions. They have found that the number of fatal cancers was less than or equal to 2 per 1 million children and it was higher for the age of 0.5-2 years for all 3 positions of skull radiographs which have good agreement with our research findings. The attributed lifetime cancer mortality risk for children is higher because they have a larger proportion of dividing cells due to their grows periods, therefore, they are inherently more vulnerable to radiation. In another study, Ronckers et al. (40) evaluated the cancer mortality in a cohort study of 5573 females with scoliosis and other spine disorders diagnosed between 1912 and 1965. The cancer mortality was reported $8 \%$ higher than expected, and also the breast cancer mortality rate was higher compared to the other cancers (lung, liver, and cervical). In our study, the breast cancer mortality risk was higher than most of the other cancers like liver, colon, uterus, and ovary, but it was lower than lung cancer mortality risk. This difference may cause by different exposure parameters and the model of cancer risk estimation.

Literature suggest that changing image orientation from AP view to PA view could greatly decrease the organ dose by approximately three-to eight-fold for some sensitive organs like breast and thyroid. Because in PA position the absorbed radiation in theses organs is much lower $(7,41)$. Thus, in the current study, the increment of cancer risks such as ovary, bladder, liver, and colon can be explained by higher rate of AP positions radiographies instead of PA views in some examinations, which also included the high frequency examinations like lumbar, pelvis, and abdomen.

Lifetime cancer incidence and mortality risks induced by irradiations from digital radiographies are strongly correlated (inverse correlation) with age at exposure time in both genders. The correlations can be found in figure 2. Other studies report the same correlations between age and cancer incidence risks (17-20). Higher risks in lower age of exposure, may be due to higher lifetime span and increasing the chance of cancer occurring. Most of the cancers have long latent period, and longer lifetime time span is closely related to a higher chance of lifetime cancer incidence.

One of the limitations of this study was the use of patient data belonging to a certain time period. In addition, the models of imaging

Int. J. Radiat. Res., Vol. 18 No. 4, October 2020 
systems and calibration procedure are the factors which can cause unwanted variation in the results.

This study can be useful for specialists in radiation protection and radiology in order to familiarize them with cancer risks of annual radiography in a big population. Through monitoring patient safety, quality control, quality assurance of radiology machines, and choosing appropriate imaging parameters, the effective dose and the number of excessive cancers due to the common radiographies would be decreased.

\section{CONCLUSION}

Our findings demonstrated that there are low but significant risks of cancer incidence for patients undergoing digital radiographies, which included a large percentage of population in Mazandaran provience. The total cancer risk values in digital radiography examinatins for females and children were higher compared to males and adults, however, the cancer risks were in the acceptable range regarding previous studies. Therefore, further efforts like appropriate patient setup and beam geometry should be carried out to decrease patient doses.

\section{ACKNOWLEDGMENT}

This article was supported and approved by Babol University of Medical Sciences (Babol, Iran) with the registration number of "MUBABOL.HRL.REC.1395.86". The authors would like to thank the radiography centers for their sincere collaboration in this project.

\section{Conflicts of interest: Declared none.}

\section{REFERENCES}

1. Tarighatnia A, Mohammadalian A, Ghojazade M, Pourafkari L, Farajollahi A, Beam projections (2017) radiation expo- sure in transradial and transfemoral approaches during coronary angiography. Anatol J Cardiol, 18(4): 298-303.

2. Tarighatnia A, Alian AHM, Ghojazadeh M, Farajollahi AR (2016) Comparison of the patient radiation exposure during coronary angiography and angioplasty procedures using trans-radial and trans-femoral access. J Cardiovasc Thorac Res, 8(2): 77-82.

3. Tarighatnia A, Pourafkari L, Farajollahi A, Mohammadalian AH, Ghojazadeh M, Nader ND (2018) Operator radiation exposure during transradial coronary angiography. Herz, 43(6):535-542.

4. Hoseini Motlagh Z, Shabestani Monfared A, Deevband MR, Abedi-Firouzjah R, Ghaemian N, Abdi R, et al. (2020) Determination of Diagnostic Reference Level in Routine Examinations of Digital Radiography in Mazandaran Province. Radiat Prot Dosim, 190(1): 31-37

5. Le Coultre R, Bize J, Champendal M, Wittwer D, Ryckx N, Aroua A, et al. (2016) Exposure of the Swiss population by radiodiagnostics: 2013 review. Radiat Prot Dosim, 169/14): $221-224$.

6. Zhang M and C Chu (2012) Optimization of the radiological protection of patients undergoing digital radiography. J Digit Imaging, 25(1): 196-200.

7. Hui SC, Pialasse J-P, Wong JY, Lam T, Ng BK, Cheng JC, et al. (2016) Radiation dose of digital radiography (DR) versus micro-dose X-ray (EOS) on patients with adolescent idiopathic scoliosis: 2016 SOSORT-IRSSD "John Sevastic Award" Winner in Imaging Research. Scoliosis Spinal Disord, 11(1): 46.

8. Omidvar F, Bouzarjomehri F, Falahati F, Zare MH (2020) Patient radiation dosimetry during interventional cardiac procedures. Int J Radiat Res, 18(3): 511-519.

9. Shahbazi-Gahrouei $D$ and Baradaran-Ghahfarokhi $M$ (2013) Assessment of entrance surface dose and health risk from common radiology examinations in Iran. Radiat Prot Dosim, 154(3): 308-313.

10. Aliasgharzadeh A, Shahbazi-Gahrouei D, Aminolroayaei $F$ (2018) Radiation cancer risk from doses to newborn infants hospitalized in neonatal intensive care units in children hospitals of Isfahan province. Int J Radiat Res, 16(1): 117-122.

11. Publication 93, ICRP (2004) Managing patient dose in digital radiology. ICRP. Ann ICRP. 34(1).

12. Omrane LB, Verhaegen F, Chahed N, Mtimet S (2003) An investigation of entrance surface dose calculations for diagnostic radiology using Monte Carlo simulations and radiotherapy dosimetry formalisms. Phys Med Biol, $\mathbf{4 8}$ (12): 1809.

13. Bouzarjomehri F and Tsapaki V (2010) Evaluation of cancer risk of the patients undergoing coronary angiography in Yazd, Iran. Int J Radiat Res, 8(3): 161-167.

14. Paydar R, Takavar A, Kardan MR, Babakhani A, Deevband MR, Saber S (2012) Patient effective dose evaluation for chest $\mathrm{X}$-ray examination in three digital radiography centers. Int J Radiat Res, 10(3/4): 139-143. 


\section{Hosseini et al. / Estimating the cancer risk in digital radiography}

15. Mohsenzadeh B, Deevband M, Paydar R, Ghorbani M (2020) Assessment of patient dose in routine digital radiography in Iran. Int J Radiat Res, 18(3): 449-460.

16. Janbabanezhad-Toori A, Deevband MR, ShabestaniMonfared A, Abdi R, Nabahati M (2016) Pediatric dose assessment in common $\mathrm{CT}$ examination towards establishment of related regional DRL in Mazandaran, Iran. Int J Radiat Res, 14(3): 251-256.

17. Abedi-Firouzjah R, Vafapour H, Banaei A, Gorji KE, Najafzadeh M, Ataei G, et al (2020) Data on the cancer risk and mortalities induced by annual background radiations at various ages in Kohgiluyeh and Boyer-Ahmad province, Iran. Data in Brief, 30: 105487.

18. Mirdoraghi M, Banaei A, Asl JF (2018) Asl, Data on the estimating the risk of cancer due to some common radiographs in Tehran city. Data in brief, 20:1269-1273.

19. Wrixon AD (2008) New ICRP recommendations. J Radiat Prot Res, 28(2): 161.

20. National Research Council (2006) Health risks from exposure to low levels of ionizing radiation: BEIR VII phase 2 Vol. 7. National Academies Press.

21. Chaparian A and Dehghanzade F (2017) Evaluation of radiation-induced cancer risk to patients undergoing intra-oral and panoramic dental radiographies using experimental measurements and Monte Carlo calculations. Int J Radiat Res, 15(2): 197-205.

22. Bahreyni Toossi MT and Zare H (2005) Organ and effective dose arising from conventional diagnostic X-ray examinations by Monte Carlo simulation (MCNP-4C Code). Int J Radiat Res, 3(1): 21-30.

23. Bouzarjomehri $F$ (2004) Patient dose in routine X-ray examinations in Yazd state. Int J Radiat Res, 1(4): 199-204.

24. Khoshdel-Navi D, Shabestani-Monfared A, Deevband MR, Abdi R, Nabahati M (2016) Local-reference patient dose evaluation in conventional radiography examinations in mazandaran, Iran. JBPE, 6(2): 61-70.

25. Bijari S, Banaei A, Kanani M, Kanani M (2016) Assessment of Entrance Skin Doses and Effective Dose for Common Xray Diagnostic Examinations. J Paramed Sci, 11(3): 1-5.

26. Ebrahimnejad Gorji K, Abedi Firouzjah R, Khanzadeh F, Abdi-Goushbolagh N, Banaei A (2019) Estimating the Absorbed Dose of Organs in Pediatric Imaging of 99mTcDTPATC-DTPA Radiopharmaceutical using MIRDOSE Software. JBPE, 9(3): 285-294.

27. Khorramian D, Sistani S, Banaei A, Bijari S (2017) Estimation and assessment of the effective doses for radiosensitive organs in women undergoing chest CT scans with or without automatic exposure control system. TUMJ, 75(7): 496-503.

28. Bernasconi A, Bernasconi N, Natsume J, Antel SB, Andermann F, Arnold DL (2003) Magnetic resonance spectroscopy and imaging of the thalamus in idiopathic generalized epilepsy. Brain, 126(11): 2447-2454.

29. Changizi V, Azarakhsh F, Pooladi M (2015) Evaluation of the role of metabolites in the diagnosis of the brain tumors using the MRS of the intensified nuclear magnet. $J$ Paramed Sci, 6(2): 72-78.

30. Zhang M and Chu C (2012) Optimization of the radiological protection of patients undergoing digital radiography. $J$ digit imaging, 25(1): 196-200.

31. The American Association of Physicists in Medicine (AAPM) (1977) Basic Quality Control in Diagnostic Radiology. Report No. 4.

32. The American Association of Physicists in Medicine (AAPM) (2002) Quality Control in Diagnostic Radiology. Report No.74.

33. Pernicka F and McLean I (2007) Dosimetry in diagnostic radiology: an international code of practice. International Atomic Energy Agency.

34. Shabestani Monfared A, Abdi R, Saber MAA (2007) Repeat analysis program in radiology departments in Mazandaran province-Iran; Impact on population radiation dose. Int $J$ Radiat Res, 5(1): 37-40.

35. Hart D, Hiller MC, Wall B (2002) Doses to patients from medical X-ray examinations in the UK. 2000 review. National Radiological Protection Board (United Kingdom).

36. Freitas MB and Yoshimura EM (2009) Diagnostic reference levels for the most frequent radiological examinations carried out in Brazil. Rev. Panam. Salud Publica, 25: 95104.

37. Skrk D, Zontar D, Zdesar U (2006) Diagnostic reference levels for X-ray examinations in Slovenia. Radiol Oncol, $\mathbf{4 0}$ (3): 189-195.

38. Law M, Ma W-K, Lau D, Chan E, Yip L, Lam W (2016) Cumulative radiation exposure and associated cancer risk estimates for scoliosis patients: Impact of repetitive full spine radiography. Eur J Radiol, 85(3): 625-628.

39. Mazonakis M, Damilakis J, Raissaki M, Gourtsoyiannis N (2004) Radiation dose and cancer risk to children undergoing skull radiography. Pediatr Radiol, 34(8): 624-629.

40. Ronckers CM, Land CE, Miller JS, Stovall M, Lonstein JE, Doody MM (2010) Cancer mortality among women frequently exposed to radiographic examinations for spinal disorders. Radiat Res, 174(1): 83-90. 